\title{
On the approximation of local and linear radiative damping in the middle atmosphere
}

Article

Published Version

Hitchcock, P., Shepherd, T. G. and Yoden, S. (2010) On the approximation of local and linear radiative damping in the middle atmosphere. Journal of the Atmospheric Sciences, 67 (6). pp. 2070-2085. ISSN 1520-0469 doi:

https://doi.org/10.1175/2009JAS3286.1 Available at https://centaur.reading.ac.uk/31609/

It is advisable to refer to the publisher's version if you intend to cite from the work. See Guidance on citing.

To link to this article DOI: http://dx.doi.org/10.1175/2009JAS3286.1

Publisher: American Meteorological Society

All outputs in CentAUR are protected by Intellectual Property Rights law, including copyright law. Copyright and IPR is retained by the creators or other copyright holders. Terms and conditions for use of this material are defined in the End User Agreement.

www.reading.ac.uk/centaur 
Central Archive at the University of Reading

Reading's research outputs online 


\title{
On the Approximation of Local and Linear Radiative Damping in the Middle Atmosphere
}

\author{
Peter Hitchcock And Theodore G. Shepherd \\ Department of Physics, University of Toronto, Toronto, Ontario, Canada \\ SHIGEO YODEN \\ Division of Earth and Planetary Sciences, Graduate School of Science, Kyoto University, Kyoto, Japan
}

(Manuscript received 12 August 2009, in final form 13 November 2009)

\begin{abstract}
The validity of approximating radiative heating rates in the middle atmosphere by a local linear relaxation to a reference temperature state (i.e., "Newtonian cooling") is investigated. Using radiative heating rate and temperature output from a chemistry-climate model with realistic spatiotemporal variability and realistic chemical and radiative parameterizations, it is found that a linear regression model can capture more than $80 \%$ of the variance in longwave heating rates throughout most of the stratosphere and mesosphere, provided that the damping rate is allowed to vary with height, latitude, and season. The linear model describes departures from the climatological mean, not from radiative equilibrium. Photochemical damping rates in the upper stratosphere are similarly diagnosed. Three important exceptions, however, are found. The approximation of linearity breaks down near the edges of the polar vortices in both hemispheres. This nonlinearity can be well captured by including a quadratic term. The use of a scale-independent damping rate is not well justified in the lower tropical stratosphere because of the presence of a broad spectrum of vertical scales. The local assumption fails entirely during the breakup of the Antarctic vortex, where large fluctuations in temperature near the top of the vortex influence longwave heating rates within the quiescent region below. These results are relevant for mechanistic modeling studies of the middle atmosphere, particularly those investigating the final Antarctic warming.
\end{abstract}

\section{Introduction}

Radiative transfer plays an important role in damping temperature perturbations in the middle atmosphere. In general, this damping is a nonlocal process in which heat is transferred to and from remote levels of the atmosphere and the surface and radiated away to space. This process is also nonlinear, mostly as a result of the nonlinear dependence of radiated power on temperature. In the mesosphere, molecular collisions occur sufficiently infrequently that local thermodynamic equilibrium (LTE) no longer holds. A realistic representation of radiative heating in the middle atmosphere therefore requires detailed consideration of these effects.

Nonetheless, in the context of simplified (or "mechanistic") modeling studies, it is desirable to reduce the

Corresponding author address: Peter Hitchcock, Dept. of Physics, University of Toronto, 60 St. George Street, Toronto, ON M5S 1A7, Canada.

E-mail: peterh@atmosp.physics.utoronto.ca complexity of the radiative heating so that its consequences for other processes can be more easily understood. It is common, therefore, for mechanistic models to approximate radiative damping as a local linear relaxation to a reference temperature state (e.g., Holton and Mass 1976; Taguchi et al. 2001; Polvani and Kushner 2002). The time scale of this relaxation has important implications for the large-scale circulation of the middle atmosphere. In general, this damping contributes to the dissipation of wave activity (Andrews and McIntyre 1978) and thus in part mediates wave-mean flow interactions [e.g., in the driving of the quasi-biennial oscillation (QBO); (Holton and Lindzen 1972)], in addition to controlling the amplitude and structure of the waves themselves. Moreover, the magnitude of the thermal response to meridional overturning circulations induced by eddy forcings (such as gravity wave drag in the mesosphere) is proportional to the radiative damping time (Garcia and Boville 1994).

Despite the approximations involved, this "Newtonian" relaxation can describe longwave (LW) heating rates in 
the middle atmosphere surprisingly well in some cases. A significant fraction of the infrared cooling in the middle atmosphere is determined by the radiation emitted at each level that is lost directly to space (Rodgers and Walshaw 1966). Moreover, since the dominant emitter of longwave radiation in the middle atmosphere, $\mathrm{CO}_{2}$, is well mixed, the local temperature is a good predictor of heating rates. This is not the case in the troposphere, where $\mathrm{H}_{2} \mathrm{O}$ (in its vaporous and condensed states) plays a much more important role. So long as the temperature perturbations remain small, linearization of the Planck function is also justified. One purpose of this paper is to explore in some detail where and to what extent these approximations of linearity and locality hold under realistic conditions in the stratosphere and mesosphere.

Accurate estimates of appropriate radiative damping rates, however, rely on detailed radiative transfer codes. Given a reference profile of temperature and radiatively active trace gases, these are used to compute explicitly at each level $z$ the change in heating rate $\delta Q(z)$ as a result of a given temperature perturbation applied to the entire column $\delta T\left(z^{\prime}\right)$. For instance, Dickinson (1973) computed damping rates $\alpha=\delta Q(z) / \delta T$ from the 1976 U.S. Standard Atmosphere by perturbing the entire column by $0.1 \mathrm{~K}$. Using a similar approach, several authors have computed damping rates based on observed profiles of temperature and ozone (Kiehl and Solomon 1986; Gille and Lyjak 1986; Mlynczak et al. 1999). The computed rates show a strong dependence on latitude and season. This dependence can be partially explained by the temperature dependence of the derivative of the Planck function, although the temperature dependence of the transmission functions is also potentially important.

An important consequence of the nonlocal nature of the radiative damping is that thermal perturbations with short vertical length scales are damped more rapidly than those with longer length scales. Fels (1982) showed that under Wentzel-Kramers-Brillouin (WKB)-like assumptions that are generally well satisfied in the middle atmosphere, wavelike disturbances are linearly damped at a rate $\alpha(z, n)$ dependent on the local vertical wavelength $2 \pi / n$. They are computed simply by specifying a perturbation $\delta T\left(z^{\prime}\right)=\epsilon \cos \left[n\left(z-z^{\prime}\right)\right]$, with amplitude $\epsilon$. In this framework, the damping rates computed by the studies mentioned above correspond to $n=0$. This scale-dependent parameterization has been updated to include non-LTE effects in the mesosphere (Fels 1984), as well as more recent estimates of the quenching rate of $\mathrm{CO}_{2}$ by atomic oxygen (Zhu 1993) and the effect of curvature in the background temperature profile (Bresser et al. 1995).

Shortwave (SW) radiation provides another source of radiative damping due to the dependence of ozone mixing ratios on temperature (Craig and Ohring 1958). In photochemical equilibrium, higher temperatures give rise to reduced ozone abundances. This reduces shortwave absorption, leading to the damping of temperature perturbations where ozone is under photochemical control. Where ozone is under dynamical control, on the other hand, eddy transport tends to lead to positive correlations between ozone and temperature (Hartmann 1981). As a result, in the lower summer stratosphere, shortwave heating can destabilize certain linear modes (Nathan et al. 1994). Ozone perturbations also have a significant, nonlocal "opacity" effect as they allow more or less shortwave radiation to penetrate to lower levels. This opacity effect gives rise again to a dependence on the vertical scale of the temperature perturbations (Haigh 1985). In general, the complexity of these effects leads to weaker linear correlations between temperatures and shortwave heating rates than between temperatures and longwave cooling rates; nevertheless, in some cases the local effects are sufficiently dominant that an effective damping rate can be defined.

A number of studies have taken a more empirical approach to specifying the temperature perturbations being damped. In this approach, heating rates are computed offline from observed (or reanalyzed) temperature and trace gas profiles, and effective damping rates are then defined by linear regression. Ghazi et al. (1985) used satellite retrievals of temperatures and ozone mixing ratios at polar latitudes on selected days with strong planetary waves, finding reasonable agreement with the longwave damping computed by Dickinson (1973). Pawson et al. (1992) extended this study to satellite observations of the whole middle atmosphere for several months' worth of data. Using 17 years of National Centers for Environmental Prediction daily analyses, Newman and Rosenfield (1997) demonstrated that such a linear regression model can capture a large fraction of the heating rate variance throughout the stratosphere, provided that damping rates are allowed to vary with height, season, and latitude.

We use a similar approach here to investigate the relationship between the temperature and radiative heating rate fields produced by the Canadian Middle Atmosphere Model (CMAM), a chemistry-climate model (CCM) with detailed radiative parameterizations and a lid near $100 \mathrm{~km}$, encompassing the entire middle atmosphere. The use of a comprehensive model with external forcings held fixed allows us to attribute disparities between the statistical fit and the model data to the radiative response, as opposed to measurement uncertainties or secular changes in the real atmosphere. We are also able to obtain a relatively smooth function to describe the damping rates as a result of the improved statistics. 
We find that the assumptions of locality and linearity do hold to a good approximation for longwave damping rates, even in the mesosphere where non-LTE effects become important. We find two notable exceptions where nonlocal effects become important, and one region in which nonlinear effects are important. First, in the lower tropical stratosphere, the spectrum of perturbations to the vertical temperature profile is sufficiently broad that scaledependent effects present significant difficulty in defining a single effective damping rate. Second, during the latespring breakup of the Antarctic vortex, strong temperature changes at the top of the decaying vortex dominate the radiative response to the weak local temperature changes in the vortex core below. This nonlocal effect cannot be described by the scale-dependent framework of Fels (1982). The assumption of linearity fails only near the edge of the polar vortex where temperature variations are large. These assumptions also hold for shortwave heating rates where ozone is under strong photochemical control, although the damping rates are a strong function of the zenith angle, which somewhat complicates the analysis.

This paper is organized as follows: In the next section, the CMAM and its radiative parameterizations are briefly described. We then turn to longwave damping rates, briefly presenting some radiative transfer theory that is useful for interpreting the results, which we then present. Results for the shortwave damping rates are discussed in the subsequent section, after which the paper concludes with a linear estimate of the radiativephotochemical equilibrium temperature and a discussion of the implication of these results for mechanistic modeling studies.

\section{Model data}

The CMAM is an extension of the Canadian Centre for Climate Modeling and Analysis (CCCma) tropospheric general circulation model (Scinocca et al. 2008), including a comprehensive set of physical parameterizations and a detailed representation of stratospheric chemistry (de Grandpré et al. 2000). The model has been validated against observations and other CCMs as part of the CCM validation activity (Eyring et al. 2006). The configuration of the model used here is similar to that submitted to the first round of model intercomparisons (CCMVal-1), in which CMAM was found to be among the best-performing of the models in terms of agreement with observations, according to the processoriented grading exercise of Waugh and Eyring (2008).

The radiation scheme employed by the CMAM is described in detail elsewhere (Fomichev et al. 1998, 2004;
Fomichev and Blanchet 1995 and references therein). We briefly mention the radiative species and processes taken into account by the parameterizations and refer readers interested in further details to the references given above. The longwave scheme used in the middle atmosphere includes the $15-\mu \mathrm{m}$ carbon dioxide band, the 9.6- $\mu \mathrm{m}$ ozone band, and the far-infrared rotational band of water vapor and accounts for the breakdown of LTE in the mesosphere. The tropospheric scheme includes $\mathrm{H}_{2} \mathrm{O}$, $\mathrm{CO}_{2}, \mathrm{O}_{3}, \mathrm{CH}_{4}, \mathrm{~N}_{2} \mathrm{O}, \mathrm{CFC11}$ and $\mathrm{CFC12}$, clouds, and aerosols. The shortwave radiation scheme includes effects of absorption by $\mathrm{O}_{3}, \mathrm{H}_{2} \mathrm{O}, \mathrm{CO}_{2}$, and $\mathrm{O}_{2}$ and includes chemical heating, sphericity, and non-LTE effects in the near-infrared absorption by $\mathrm{CO}_{2}$.

Four time slice runs of 40 model years were examined here. These runs included a dynamics-only configuration with prescribed ozone and sea surface temperatures (SSTs), an interactive chemistry configuration with prescribed SSTs, a coupled ocean configuration with prescribed ozone, and finally a configuration with a coupled ocean and interactive chemistry. All runs had a $\mathrm{CO}_{2}$ mixing ratio of $348 \mathrm{ppm}$. Most results presented below are from the interactive chemistry run with fixed SSTs, though the fully coupled run gave very similar results. The effects of interactive chemistry are briefly discussed, although attributing the changes in damping rates between the interactive and noninteractive runs is difficult since the predicted ozone climatology is significantly different from the prescribed climatology (and consequently the temperature climatology is significantly different as well).

\section{Longwave relaxation rates}

\section{a. Theory}

All of the effective damping rates reported in this study have been computed using linear regression techniques. To gain some insight into these empirical results, we briefly review some relevant radiative transfer theory. Following Andrews et al. (1987), the longwave heating rate due to a given species of absorber from a spectral band $r$ can be written in the exchange integral formulation

$$
\begin{aligned}
h_{r}(z)= & -\frac{\pi m_{a} k_{r}}{c_{p}}\left\langle\int_{z>z^{\prime}}^{1}\left\{B_{r}\left[T\left(z^{\prime}\right)\right]-B_{r}[T(z)]\right\} d \Gamma_{r}\left(z, z^{\prime}\right)\right. \\
& \left.+\int_{z<z^{\prime}}^{1}\left\{B_{r}\left[T\left(z^{\prime}\right)\right]-B_{r}[T(z)]\right\} d \Gamma_{r}\left(z, z^{\prime}\right)\right\rangle
\end{aligned}
$$


where $m_{a}$ is the mass mixing ratio of the absorbing species, $c_{p}$ is the specific heat at constant pressure, $k_{r}$ is the absorption coefficient, $B_{r}$ is the Planck function, and the subscript $r$ indicates a band-averaged quantity. The escape function $\Gamma_{r}\left(z, z^{\prime}\right)$ is a normalized first derivative of the transmission function, and for $z^{\prime}>z$ or $z^{\prime}<z$ gives the probability that a photon emitted upward or downward (respectively) at $z$ reaches $z^{\prime}$ prior to being absorbed. Note that $\Gamma_{r}\left(z, z^{\prime}\right)$ provides a convenient coordinate system (for every $z$ ) in which the exchange of radiation between levels of the atmosphere is emphasized over details of the radiative transfer computation. The terms representing cooling to space and exchange with the surface are included in the integrals by setting $B_{r}\left[T\left(z^{\prime}\right)\right]$ to 0 where $\Gamma_{r}\left(z, z^{\prime}\right) \leq \Gamma_{r}(z, \infty)$ and $z^{\prime}>z$, and to the flux density emitted by the surface $B_{r}\left(T_{g}\right)$ where $\Gamma_{r}\left(z, z^{\prime}\right) \leq$ $\Gamma_{r}(z, 0)$ and $z^{\prime}<z$.

Most radiation emitted by the $15-\mu \mathrm{m}$ carbon dioxide band is exchanged between nearby levels so that the escape functions are strongly peaked (Leovy 1984). If the temperature profile varies weakly on this radiative length scale, it is valid to expand the integrand of (1) as a Taylor series in $\left(z^{\prime}-z\right)$. The Planck function and its derivatives can then be taken out of the integrals, and we can write (1) in terms of moments of the effective radiation lengths

$$
\ell_{m}^{u, d}=\int_{0}^{1}\left|z^{\prime}-z\right|^{m} d \Gamma\left(z, z^{\prime}\right)
$$

where the superscripts indicate whether the integral is taken above $(u)$ or below $(d)$ level $z$ :

$$
\begin{aligned}
h_{r}(z)= & \frac{\pi m_{\mathrm{CO}_{2}} k_{r}}{c_{p}}\left\{-B_{r}[T(z)] \Gamma(z, \infty)+\left(\ell_{1}^{u}-\ell_{1}^{d}\right) \frac{d B_{r}}{d T} \frac{d T}{d z}\right. \\
& \left.+\frac{1}{2}\left(\ell_{2}^{u}+\ell_{2}^{d}\right) \frac{d B_{r}}{d T} \frac{d^{2} T}{d z^{2}}+\cdots\right\} .
\end{aligned}
$$

The derivative of the first term in this expansion is sometimes called the Newtonian cooling coefficient. More generally, the (scale dependent) change in the local heating rate $\delta h_{r}(z)$ associated with a temperature perturbation $\delta T=\epsilon \cos \left[n\left(z-z^{\prime}\right)\right]$ can be computed in this context.

One can posit three relevant length scales: that of the background temperature profile $L_{c}$, of the temperature perturbation $L^{\prime}$, and of the radiation $L_{r}$. We take the ratio of $L_{r}$ and $L^{\prime}$ to be $O(1)$ and define a nondimensional number $\mathrm{Ln}=L_{r} / L_{c}$. We further define Tm, the ratio of the temperature perturbation to the background temperature. Retaining terms to $O\left(\mathrm{LnTm}^{2}\right)$ and $O\left(\mathrm{Ln}^{2} \mathrm{Tm}\right)$, the damping rate is

$$
\begin{aligned}
\alpha(z, n)= & -\frac{\delta h_{r}(z)}{\delta T(z)}=\frac{\pi m_{\mathrm{CO}_{2}} k_{r}}{c_{P}}\left\langle\frac{d B_{r}}{d T}[\Gamma(z, \infty)\right. \\
& \left.+\frac{1}{2}\left(\ell_{2}^{u}+\ell_{2}^{d}\right) n^{2}\right]+\frac{d^{2} B_{r}}{d T^{2}}\left\{\frac{d T}{d z}\left(\ell_{1}^{d}-\ell_{1}^{u}\right)\right. \\
& \left.\left.+\left[\frac{1}{2} \Gamma(z, \infty)+\frac{1}{2}\left(\ell_{2}^{u}+\ell_{2}^{d}\right) n^{2}\right] \delta T\right\}+\cdots\right\rangle .
\end{aligned}
$$

The $n^{2}$ dependence on the vertical wavenumber is recovered as a consequence of the radiative diffusion term. The expansion breaks down for large $n$ as the characteristic length scale of the temperature profile becomes shorter than the effective radiation length scale. Sasamori and London (1966) showed that in this limit damping rates vary as $n^{0.5}$, although the dependence saturates in the mesosphere as a result of non-LTE processes (Fels 1984).

The damping rates depend on the background temperature primarily through the first derivative of the Planck function. They (and their scale dependency) will also tend to increase with height as the radiation length scales increase.

Corrections to the linear damping rate arise at higher order in Ln as a result of the lapse rate and curvature of the background temperature profile. In our scaling the latter enters only at $O\left(\mathrm{Ln}^{3} \mathrm{Tm}\right)$ and has been dropped, but it may be of equal importance to the lapse rate correction because of cancellations between $\ell_{1}^{u}$ and $\ell_{1}^{d}$ (Bresser et al. 1995). Nonlinearities in this expression are a result of the curvature of the Planck function and arise at $O\left(\mathrm{LnTm}^{2}\right)$.

Terms proportional to odd powers of odd derivatives of the temperature will force a change in the vertical phase of the temperature perturbation; these too tend to be small in the middle atmosphere as a result of cancellations between the odd moments of the upward and downward radiation lengths (Fels 1984).

This expression [(3)] neglects the temperature dependence of the absorption coefficients (and thus the escape function). Other studies have reported that they contribute no more than $20 \%$ of the total change in heating rates (Zhu 1993), although to our knowledge no systematic investigation of their role in determining the damping of large-scale perturbations has been carried out. The expression can also be used for the 9.6- $\mu \mathrm{m}$ band of ozone, though the effective radiation lengths will generally be longer, and in the lowermost stratosphere exchange with the surface (or cloud tops) dominates. The expression also neglects the temperature dependence of the ozone mixing ratio. Pawson et al. (1992) found some evidence that this may destabilize the lower tropical stratosphere. Although we find strong evidence for the importance of scale dependence in this region, we have not found evidence of any such radiative instability in CMAM. 
(a) (s40ac); $52 \mathrm{~N} 5 \mathrm{hPa}$ Apr 15

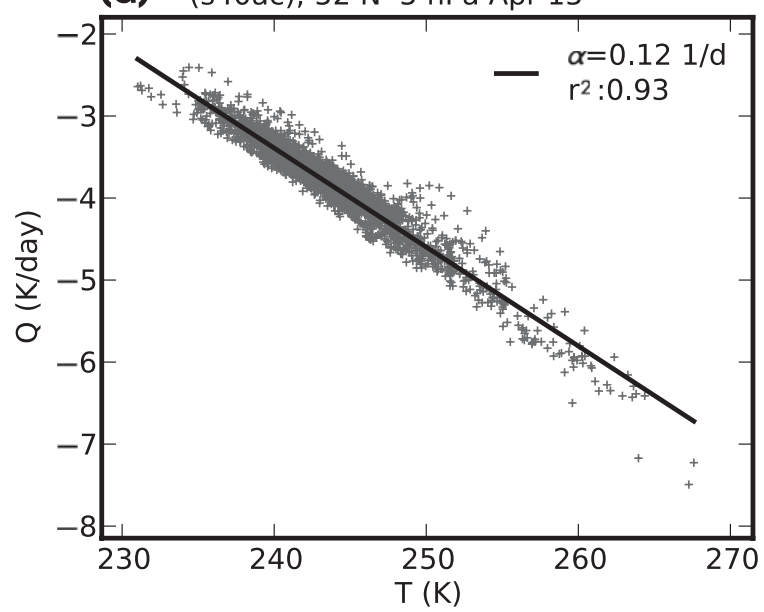

(c)

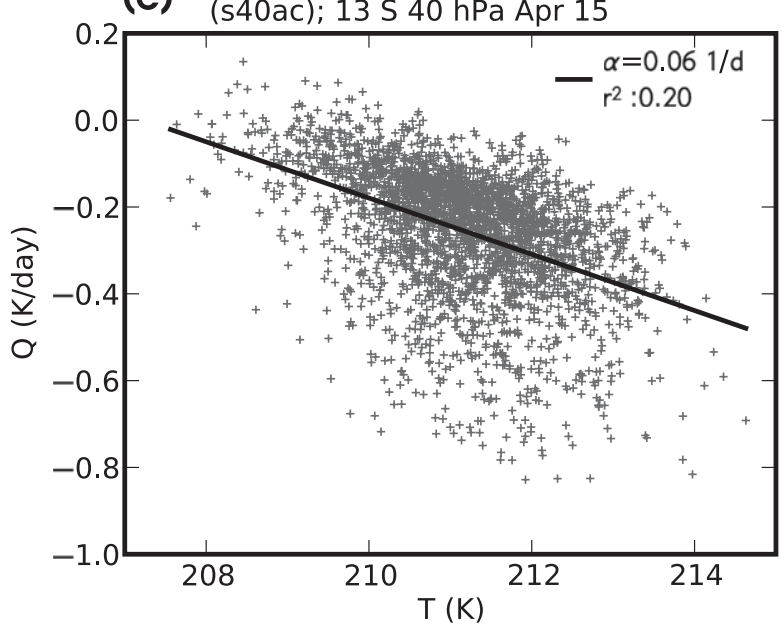

(b) (s40ac); 58 S $0.4 \mathrm{hPa}$ Apr 15

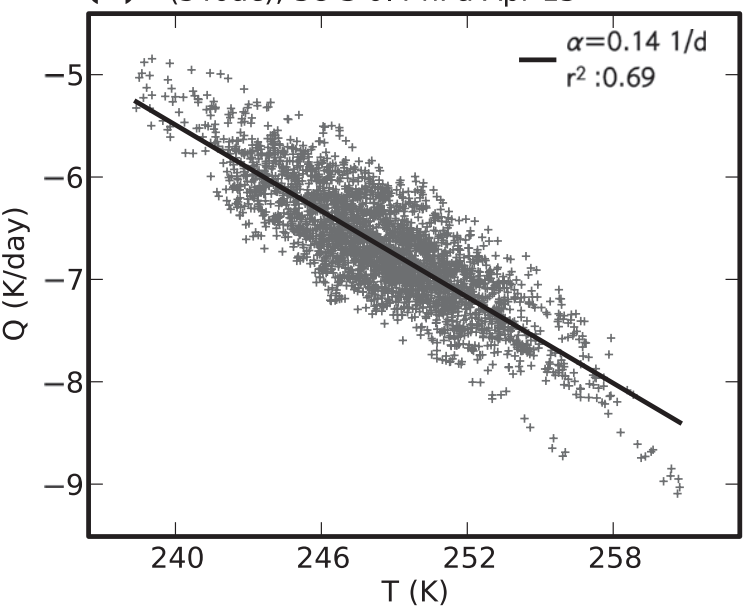

(d)

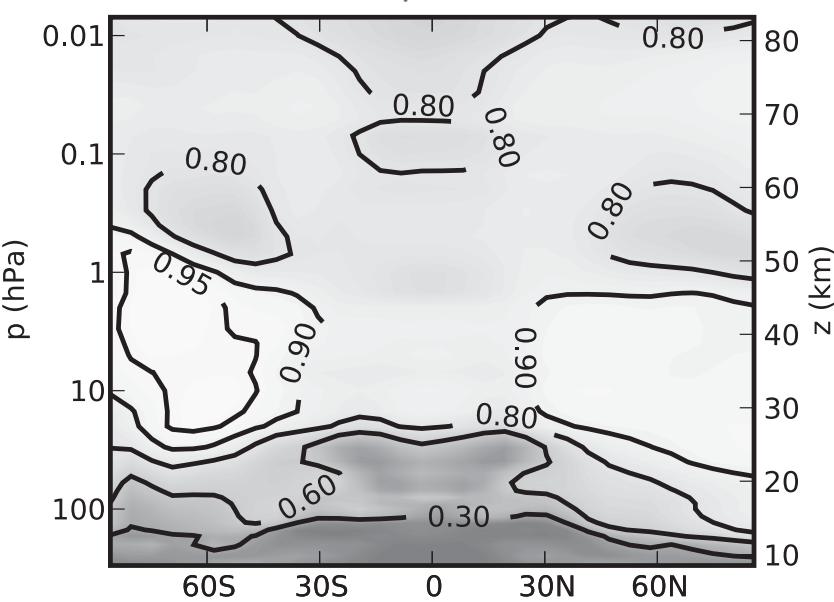

FIG. 1. Regression of longwave heating rate against temperature on $15 \mathrm{Apr}$ at (a) $52^{\circ} \mathrm{N}, 5 \mathrm{hPa}$; (b) $58^{\circ} \mathrm{S}, 0.4 \mathrm{hPa}$; and (c) $13^{\circ} \mathrm{S}, 40 \mathrm{hPa}$. The effective damping rate and variance explained are indicated in the legend. (d) Fraction of variance in longwave heating rates explained by local temperature anomalies. The monthly mean for April is shown.

To complete a theory of longwave damping rates in the middle atmosphere, we would need a radiative transfer model to compute the escape functions, as well as a theory for the structure of the temperature profile and typical perturbations. We proceed instead in an empirical fashion by using temperatures and radiative heating rates generated by a CCM. We define the background state as the zonal and climatological mean (denoted by square brackets and a subscript $c$, respectively), and the perturbation as the daily mean anomaly from this state (from 6-hourly model output), $X^{\prime}=X-[X]_{c}$. Effective damping rates are then computed at every day $d, \log$-pressure height $z$, and latitude $\phi$ by linear regression:

$$
Q_{\mathrm{LW}}^{\prime}=-\alpha(d, z, \phi) T^{\prime}+\epsilon, \quad \alpha(d, z, \phi)=-\frac{\left[Q^{\prime} T^{\prime}\right]_{c}}{\left[T^{\prime} T^{\prime}\right]_{c}} .
$$

Fits are computed over all model years and longitudes, giving approximately $3 \times 10^{3}$ (nonindependent) samples at each point. The use of daily mean anomalies removes the tides. This is appropriate for mechanistic modeling studies that do not include a diurnal cycle; moreover, as discussed below, the effective damping rates agree well with tidal damping rates computed by McLandress (2002). Examples of daily fits are shown in Figs. 1a-c. Monthly averages are then performed over the fitted parameters to smooth the fields. Note that this definition of the heating rate and temperature anomalies from a climatological mean differs from that used in other studies using this approach, which use anomalies from the zonal mean. This allows us to include the damping of zonal mean perturbations, which would be complicated by the presence of secular changes in observed and reanalyzed fields. 
To the extent that linear superposition can be applied, this effective damping rate can be related to the scaledependent rates by decomposing the anomalies into vertical spectral components $T_{n}^{\prime}$ and $Q_{n}^{\prime}$. The effective damping rate can be seen as an average, weighted by the fraction of the total power in each wavenumber:

$$
\alpha(d, z, \phi)=-\sum_{n} \frac{\left[Q_{n}^{\prime *} T_{n}^{\prime}\right]_{c}}{\left[T^{\prime} T^{\prime}\right]_{c}}=\sum_{n} \alpha(d, z, \phi, n) \frac{\left[T_{n}^{* *} T_{n}^{\prime}\right]_{c}}{\left[T^{\prime} T^{\prime}\right]_{c}},
$$

where the asterisk denotes complex conjugation. While conceptually useful, this decomposition would need to be local in nature to be used quantitatively. This argument can also be extended to predict the correlation coefficient of the fit, although we will show that the presence of perturbations at a variety of different scales is not the only reason the fit breaks down.

Figure 1d shows the variance explained as a function of latitude and height for the month of April. In general, above the tropopause, the statistical model fits quite well. In the mid to upper stratosphere, the local, linear approximation captures nearly all of the variance of the longwave heating rates with squared correlations greater than 0.9 in the extratropics (e.g., Fig. 1a) and somewhat lower correlations in the tropics. The quality of the fit drops off somewhat in the mesosphere, with minima in the extratropical lower mesosphere (e.g., Fig. 1b) and near the stratopause and mesopause in the tropics. In the extratropical lower stratosphere where the temperature varies comparatively little, the fit still captures greater than $60 \%$ of the variance.

However, in the tropical lower stratosphere the fit breaks down significantly. Figure $1 \mathrm{c}$ shows a scatterplot at $13^{\circ} \mathrm{S}, 40 \mathrm{hPa}$ on 15 April. The majority of points lie near the fitted line, but significantly stronger cooling rates than predicted by the linear relation occur as well. Inspection of individual model days shows that these regions of strong cooling are typically associated with synoptic-scale temperature anomalies, although the presence of high cloud tops may also play a role. These regions of low correlation shift seasonally with the climatological easterlies in these simulations, suggesting that the breaking of westward-traveling waves may play a role in generating these synoptic-scale structures.

To test for the importance of nonlinearity in the response, we include a quadratic term in a multiple regression,

$$
Q_{\mathrm{LW}}^{\prime}=Q_{0}-\alpha T^{\prime}-c T^{2}+\epsilon,
$$

with residuals $\epsilon$ and fitted parameters $Q_{0}, \alpha$, and $c$. Including a quadratic term in the fit describes an additional
$5 \%$ of the variance near the vortex edge in the mid to upper stratosphere in both hemispheres (Figs. 2a-d), as a result of the large range of temperatures found there. A roughly constant value of $0.0009 \pm 0.0003(\mathrm{~K} \text { day })^{-1}$ is found for the coefficient $c$ over this region (i.e., Figs. 2e,f). As a result of the large amount of data, the additional degree of freedom statistically improves the fit nearly everywhere. The differences are, however, physically negligible in other regions and seasons. Over the 60-K range of temperatures shown in Figs. $2 \mathrm{e}$ and $2 \mathrm{f}$, the linear damping rate varies by about $0.05 \mathrm{day}^{-1}$ - the same order as the linear rate itself-which indicates the potential dynamical importance of this nonlinearity for disturbances to the polar vortex.

\section{b. Results}

The effective damping rates are compared against the parameterization of Fels (1982) in Fig. 3 for log-pressure heights from 20 to $70 \mathrm{~km}$. The parameterized damping rates are computed from the area-weighted, monthlymean climatological temperatures in the three latitude ranges shown. Damping rates regressed from the model runs are also shown. The error bars are an estimate of the sampling error from the regression technique. They are estimated by computing effective damping rates for each nonoverlapping 5-yr period in the model runs. The error bars are then twice the standard deviation of the mean effective damping rates over the set of 5-yr periods. Profiles are shown for the tropics (Fig. 3a), southern winter midlatitudes (Fig. 3b), and Arctic winter (Fig. 3c); damping times are shown for the latter to emphasize lower stratospheric values. The regressed profiles lie within the range of parameterized rates predicted for large vertical wavelengths. Since the regressed rates are a weighted average of scale-dependent damping rates, the additional vertical structure present in the regressed rates may be a result of changes with height in the spectrum of vertical wavelengths present. Although differences may also arise from the climatological ozone profile, the time-dependent modeled ozone, or details in the radiative transfer parameterizations, this illustrates the need for a local decomposition to vertical wavenumber space (such as a wavelet transformation) in order to perform a detailed comparison with a scale-dependent parameterization such as Fels (1982). Such a transformation is beyond the scope of this work.

Monthly means of the linear damping rates computed from the quadratic fit [(6)] are shown in Fig. 4 for January, April, July, and October. The plots are shaded where the variance explained $r^{2}$ drops below 0.5 to indicate where the quality of the fit declines in each month. The broad vertical structure of low damping rates near the tropopause rising to a maximum near the 


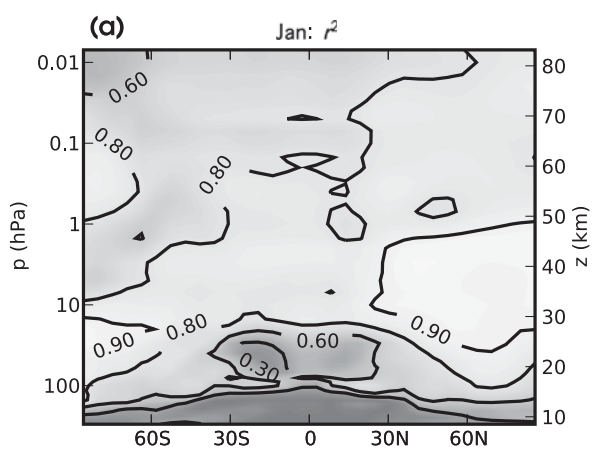

(b)

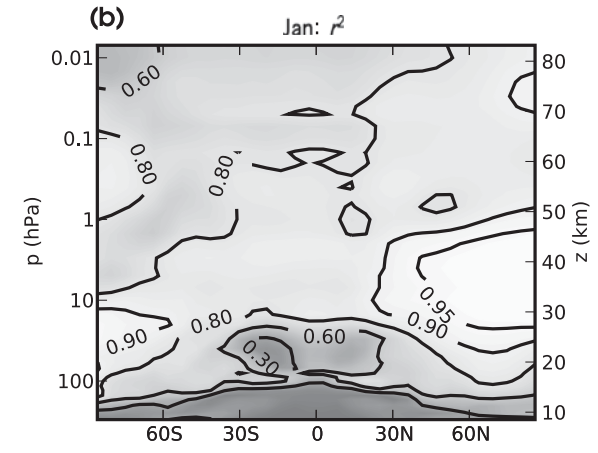

(c)

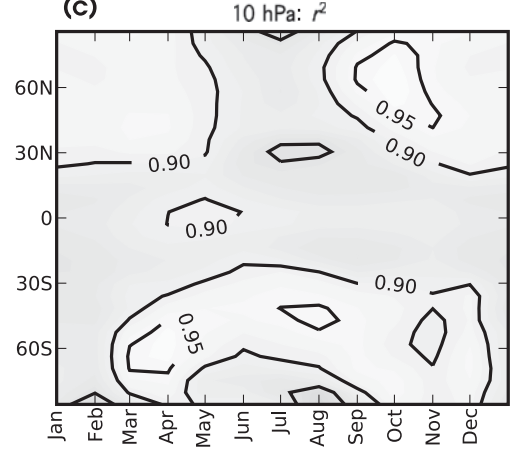

(d)

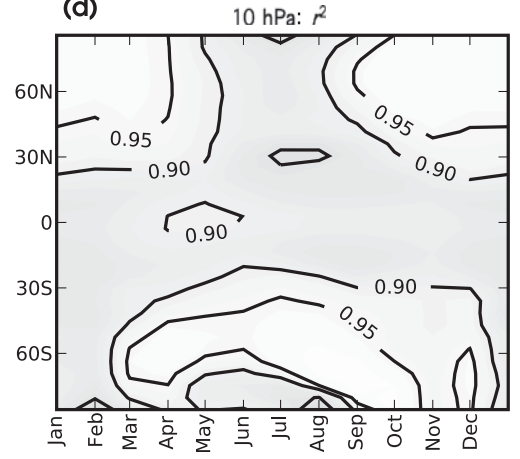

(e) (s40ac); $52 \mathrm{~S} 10 \mathrm{hPa}$ Jul 15

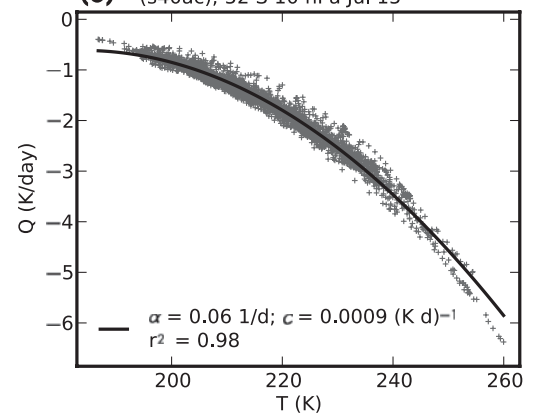

(f) (s40ac); $58 \mathrm{~N} 10 \mathrm{hPa} J a n 15$

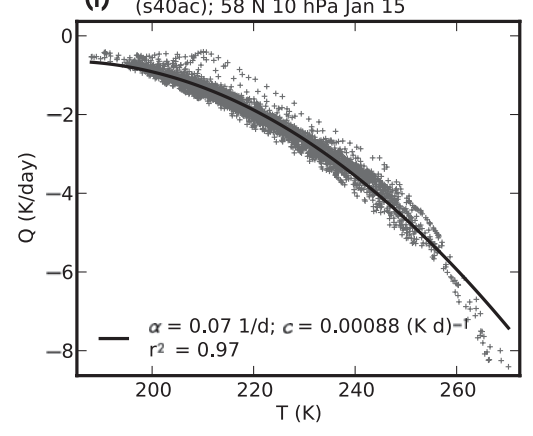

FIG. 2. (a),(b) Latitude-height plots of monthly mean $r^{2}$ for January, defined as in Fig. 1d. (c),(d) Month-latitude plots of $r^{2}$ at 10 hPa. Coefficients of determination are from regressions of the longwave heating against anomalies from the climatological temperature including in (a) and (c) a linear term only, whereas in (b) and (d) both a linear and a quadratic term are included. (e) Longwave heating rates vs temperatures on $15 \mathrm{Jul}$ at $52^{\circ} \mathrm{S}, 10 \mathrm{hPa}$. The parameters of the regression line shown are indicated in the legend. (f) As in (e), but for $15 \mathrm{Jan}$ at $58^{\circ} \mathrm{N}, 10 \mathrm{hPa}$.

stratopause and falling again in the mesosphere is apparent through most latitudes and seasons. The lowest damping rates are found near the extratropical tropopause, particularly in the winter hemisphere, despite it being warmer than the summer mesosphere. Significant meridional and seasonal variations are apparent. In the stratosphere, damping rates tend to peak in the tropics and fall off toward both poles. There is a stronger seasonal variation toward the poles, with the lowest damping rates at the base of the polar vortices.

In the mesosphere the seasonal cycle is reversed, consistent with the seasonal cycle in mesospheric temperatures. The strong peak in damping rates in the winter mesosphere above Antarctica is partly due to high temperatures but is more directly a result of the presence of a tertiary ozone maximum (Marsh et al. 2001). This feature of the ozone climatology in CMAM has not been compared in detail to observations (which are limited in this region). Nonetheless, it is notable that ozone has such a strong impact on the polar mesospheric damping rates. The asymmetry between the two hemispheres is a result of higher temperatures and ozone mixing ratios in the Antarctic winter. The increase in damping rates in the tropics toward $0.01 \mathrm{hPa}$ apparent in all seasons has also been noted in the extended CMAM (with model lid near $200 \mathrm{~km})($ McLandress 2002) and is associated with a large peak in $\mathrm{CO}_{2}$ cooling rates in the thermosphere.

The features of the seasonal cycle reported here are qualitatively similar to those reported by Kiehl and Solomon (1986) and Mlynczak et al. (1999), though the damping times computed here are not as long. The differences near the stratopause and in the extratropics are roughly $20 \%-50 \%$, but the extremely long damping times previously reported in the lower tropical stratosphere are absent here. These differences are likely due to the constant temperature perturbation used by both of these studies to compute the damping times, which would result in a larger disparity with our results near the equator as a result of the shorter vertical length scales associated with tropical waves.

The dependence of tropical damping rates $\left(20^{\circ} \mathrm{S}-20^{\circ} \mathrm{N}\right)$ on zonal wavenumber is shown in Fig. 5. This dependence is computed by filtering the anomalies prior to performing the regression. The seasonal cycle of damping rates at the equator is shown for zonal mean disturbances (Fig. 5a), for zonal wavenumbers 1-3 (Fig. 5b), and for zonal wavenumbers 4 and 5 (Fig. 5c). A small semiannual cycle is present in the lower mesosphere, consistent with 

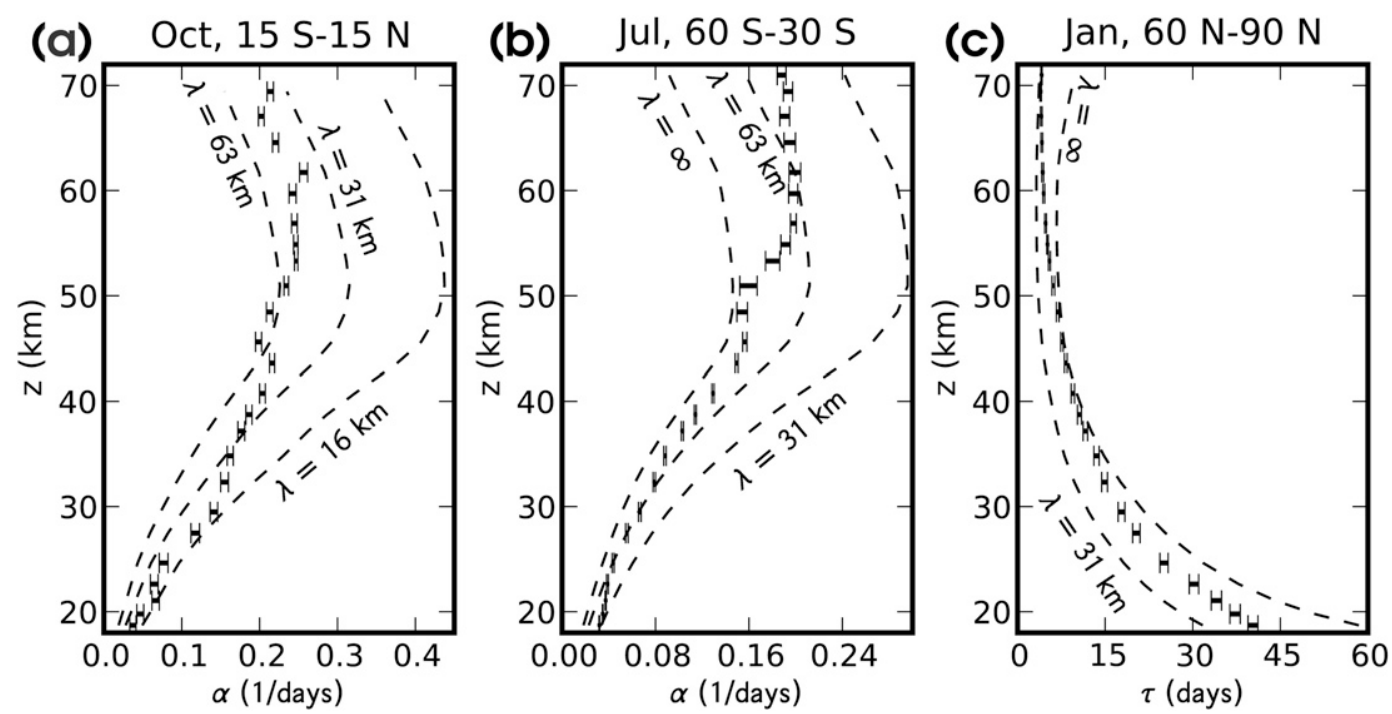

FIG. 3. Damping rates as a function of log-pressure height averaged over (a) October, from $15^{\circ} \mathrm{S}$ to $15^{\circ} \mathrm{N}$, (b) July, from $60^{\circ} \mathrm{S}$ to $30^{\circ} \mathrm{S}$, and (c) January, from $60^{\circ} \mathrm{N}$ to $90^{\circ} \mathrm{N}$. The dashed lines are computed using the parameterization of Fels (1982) with climatological temperatures taken from CMAM and vertical wavelengths $\lambda$ as labeled. The symbols indicate regressed damping rate, with error bars indicating an estimate of the sampling uncertainty (see text for details).

the timing of the semiannual oscillation in temperatures in these runs. Planetary-scale disturbances are damped significantly more strongly than zonal mean perturbations (the differences are considerably greater than the error bars estimated in Fig. 3a). The dependence on wavelength increases with height, consistent with Fels (1984). We find that the dependence on zonal wavelength is strongest in the tropics.

Turning to the polar regions where the seasonal cycle is much stronger, we find significant differences between the hemispheres (Fig. 6). The seasonal cycles of damping rates averaged poleward of $70^{\circ} \mathrm{N}$ and $70^{\circ} \mathrm{S}$ are shown in Figs. 6a and $6 \mathrm{~b}$, respectively. Enhanced damping rates are present above $0.1 \mathrm{hPa}$ during the polar night in both hemispheres, though the peak is larger in the Antarctic. Effective damping rates are small in both polar vortices because of the low temperatures but are smaller in the Antarctic, consistent with the stronger, colder vortex. However, the squared correlation coefficient for the fit differs drastically between the two hemispheres (Figs. 6c,d). While the correlation remains high throughout the year in the Arctic (with a minimum in the lowermost stratosphere during the summer months), the local relationship breaks down entirely in the Antarctic lower stratosphere (near $100 \mathrm{hPa}$ ). The fit fails within the vortex and is weakest during the spring as the vortex breaks down from above. In contrast with the Arctic, strong thermal variability in this downward-propagating region is a feature of the model's climatology (Figs. 6e,f). Within the region of strong variability the linear relationship between temperature and heating rate holds, but below it fails.
The downward influence of temperature variability at the top of the vortex is further demonstrated in Fig. 7. Figure 7a shows a scatterplot of longwave heating rates against temperature within the vortex at $50 \mathrm{hPa}, 80^{\circ} \mathrm{S}$ on 26 September of the model year. The failure of the assumption of a climatological local damping rate is evident. The clusters of points that extend with both positive and negative slope are associated with individual years, during some of which longwave radiation acts to damp perturbations but in others acts to amplify perturbations. The zonally asymmetric component of the temperature (Fig. 7b) and shortwave (Fig. 7c) and longwave (Fig. 7d) heating rates are also shown for one such year. The strong wave-1 disturbance in temperatures between 25 and $40 \mathrm{~km}$ evanesces downward into the lower stratosphere. In the region from 25 to $40 \mathrm{~km}$, the longwave heating is strongly anticorrelated with the temperatures. However, from 15 to $25 \mathrm{~km}$, as a result of the large amplitudes above, the longwave heating rates and temperatures are positively correlated. Shortwave heating rates (Fig. 7c) provide a further mechanism for downward radiative influence. From 35 to $40 \mathrm{~km}$, shortwave heating rates are also anticorrelated with the local temperature, as a result of the photochemical control of ozone abundances. Between 30 and $35 \mathrm{~km}$, however, the opacity effect (Ghazi et al. 1985; Pawson et al. 1992) leads to a positive correlation. Below about $25 \mathrm{~km}$, the heating rate remains positively correlated as a combined result of ozone transport and the opacity effect.

The heating rates induced remotely by the evanescent wave are relatively small (about $0.1 \mathrm{~K} \mathrm{day}^{-1}$ in this case, 

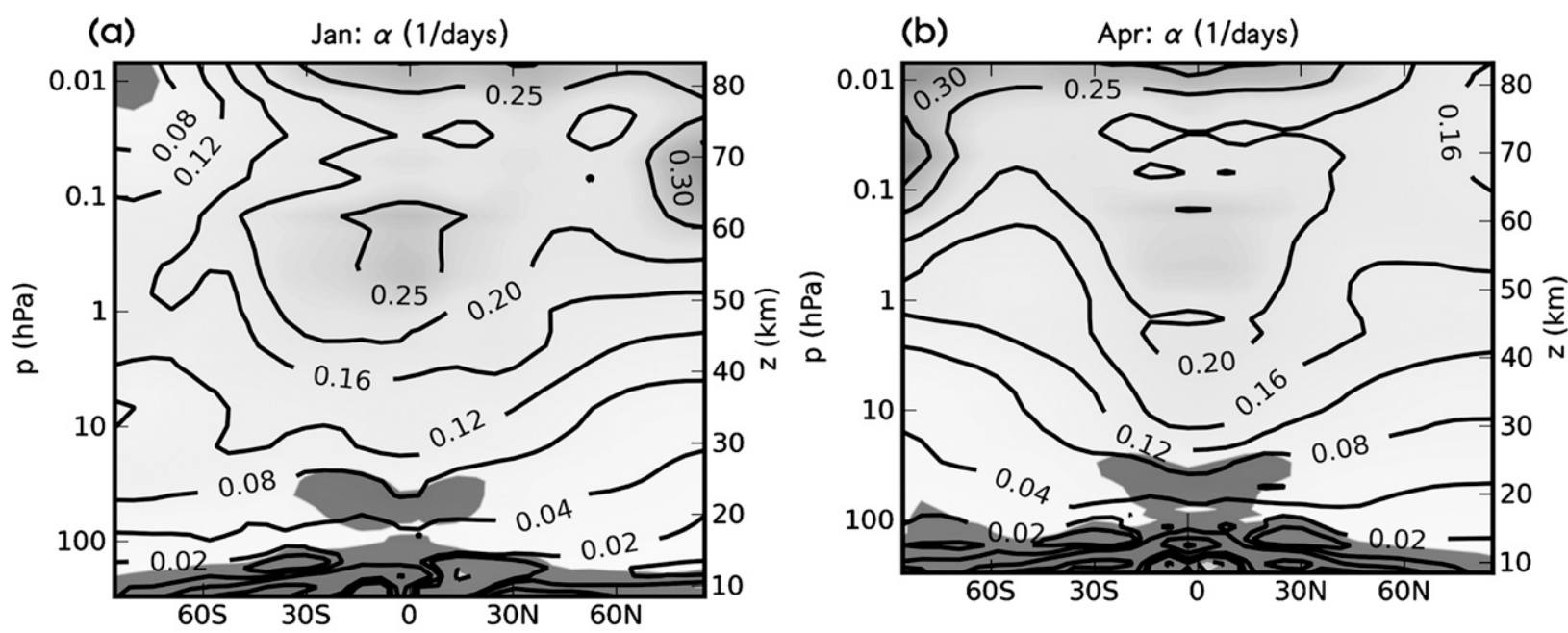

(c) Jul: $\alpha(1 /$ days $)$

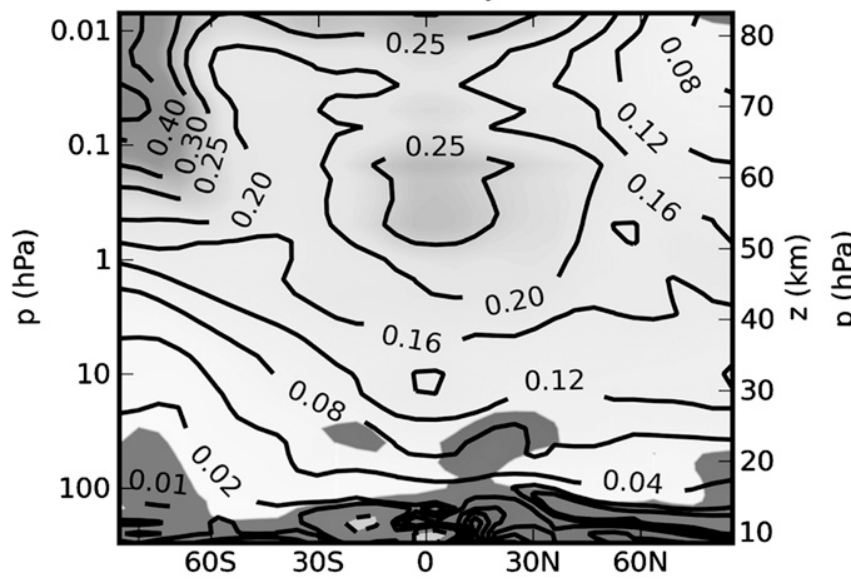

(d)

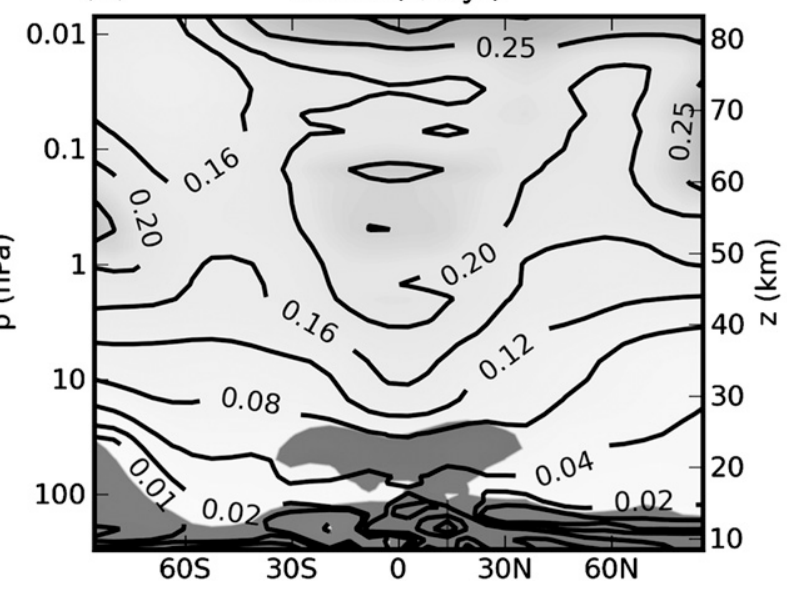

FIG. 4. Monthly mean damping rates $\left(\mathrm{day}^{-1}\right)$ for January, April, July, and October. Damping rates shown are the linear terms from a quadratic fit at each latitude, pressure level, and day. The dark shading indicates regions where the quadratic fit explains less than $50 \%$ of the variance in longwave heating rates.

which was selected for its clarity); however, they can clearly dominate the heating driven by local temperature perturbations in the Antarctic vortex. Moreover, the effective damping rates can clearly vary from year to year in this region and season (Fig. 7a), presumably as a result of the dynamical variability associated with the timing of the final warming. Although we have not demonstrated what role these nonlocal rates play in the break down of the vortex in this model, this result provides a caution against using a local, climatological
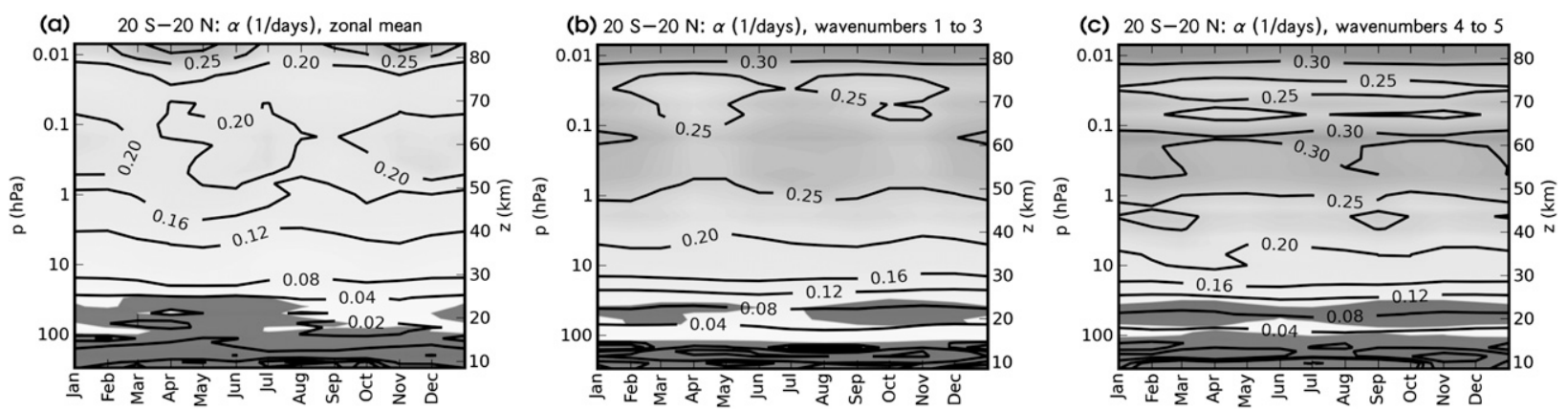

FIG. 5. Tropical monthly mean damping rates; dark shading is as in Fig. 4. Regressions were performed using (a) zonal mean anomalies only, (b) zonal wavenumbers 1-3, and (c) zonal wavenumbers 4 and 5. 

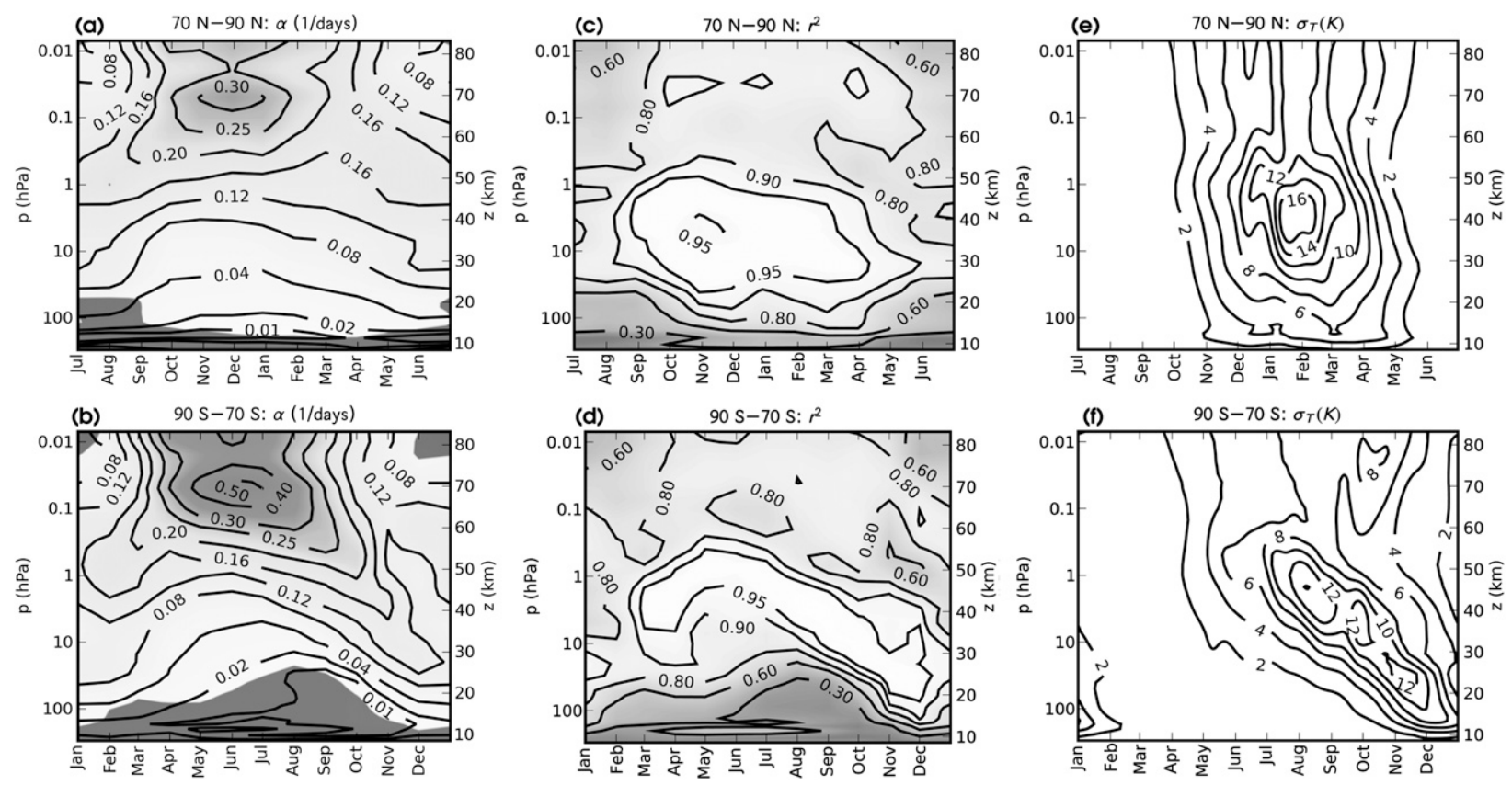

FIG. 6. (a),(b) Damping rates as a function of month and pressure, averaged over $70^{\circ}-90^{\circ} \mathrm{N}$ and $70^{\circ}-90^{\circ} \mathrm{S}$, respectively, with shading as in Fig. 4. (c),(d) Coefficients of determination for the same latitudes. (e),(f) Standard deviation of temperatures at the same latitudes. Northern Hemisphere plots are shifted by 6 months to center the winter months.

radiative damping rate in mechanistic modeling studies of this region.

\section{Shortwave relaxation rates}

A similar regression analysis can be performed on shortwave heating rate anomalies in order to diagnose effective damping rates from photochemical- and transport-related ozone perturbations (i.e., Ghazi et al. 1985). The analysis is somewhat complicated by the strong dependence of the effective damping rate on the diurnal cycle. While the regression can be performed on daily, zonal mean temperatures and heating rates, this significantly reduces the amount of data used to perform the fits and reduces their statistical significance. Instead, we diagnose this dependence directly by performing a linear regression for every day of the year as a function of the local time. Sample scatterplots are shown in Fig. 8, in the southern midlatitude spring near the stratopause at local noon (Fig. 8a) and in the sub-Arctic midstratosphere summer at 0600 local time (Fig. 8c). Where ozone is under photochemical control, there is a strong negative linear correlation between temperature and shortwave heating. Where it is controlled by transport, background ozone gradients tend to lead to a positive correlation between temperature and shortwave heating (Hartmann 1981; Nathan et al. 1994). In the latter case, the regression coefficient between temperature and shortwave heating is weaker. In both cases, a clear dependence on zenith angle is apparent (Figs. 8b,d). Error bars in the fits indicate the 95\% confidence interval of the regressed slope.

Figure 9 shows daily averages of the shortwave damping rates and coefficients of determination for the months of December (Figs. 9a,b) and June (Figs. 9c,d). A daynight mask is applied to the coefficients of determination (but not the damping rates) when performing the diurnal average. Photochemical damping is present throughout the upper stratosphere and lower mesosphere, with a broad maximum near $1 \mathrm{hPa}$ of 0.08 day $^{-1}$, falling off more quickly below than above. Somewhat larger values of 0.12 day $^{-1}$ are found above the summer pole. Significant positive correlations between temperature and shortwave heating rate are found throughout the summer lower stratosphere, with negative effective damping rates peaking at -0.04 day $^{-1}$ above the summer pole. This is consistent with the unstable, linear, westward-traveling Rossby modes found there by Nathan et al. (1994). The increased damping toward the polar region is in part a result of the length of the polar day. Similar results are seen for June, although the negative damping rates are stronger (peaking at -0.08 day $^{-1}$ ) and the damping above the pole is weaker. The correlation coefficients above the summer pole are weaker during boreal summer than during austral summer, where they maximize in December. No significant correlations were found elsewhere in the lower stratosphere, or above $0.05 \mathrm{hPa}$. 
(a) (s40ac); $80 \mathrm{~S} 50 \mathrm{hPa}$ Sep 26

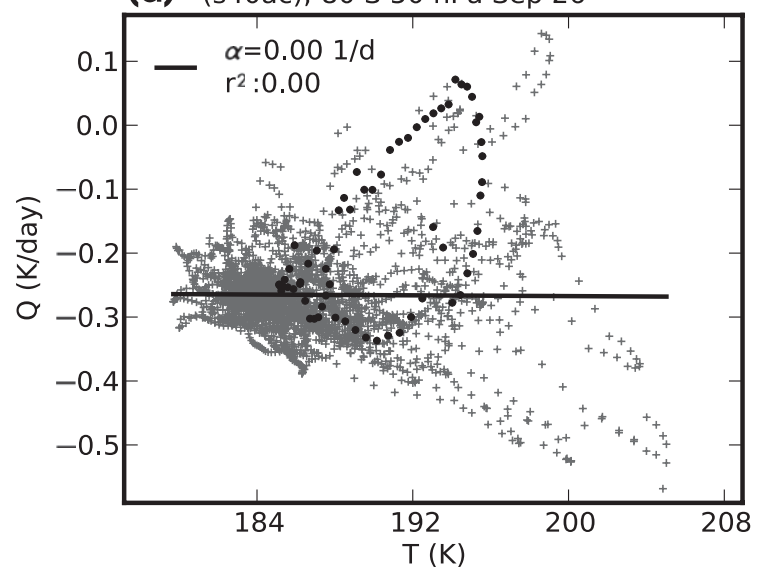

(c) (s40ac); QLw $80 \mathrm{~S}$ Sep 26 yr 38

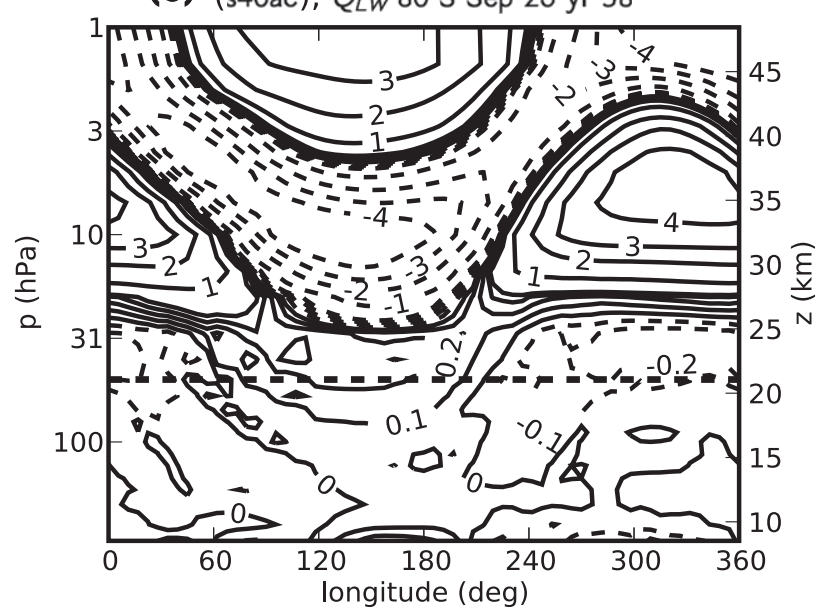

(b) $\quad(s 40 \mathrm{ac}) ; T 80 \mathrm{~S}$ Sep $26 \mathrm{yr} 38$

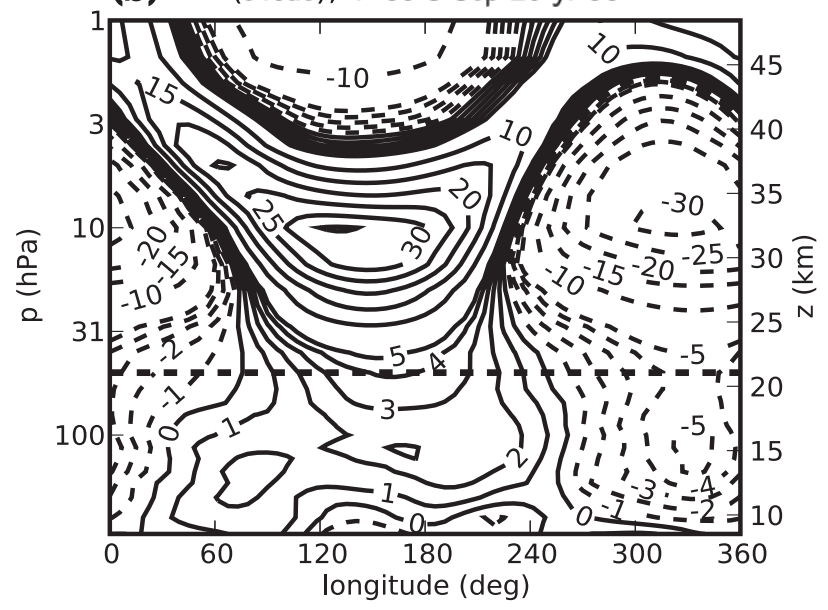

(d) (s40ac); Qsw 80 S Sep 26 yr 38

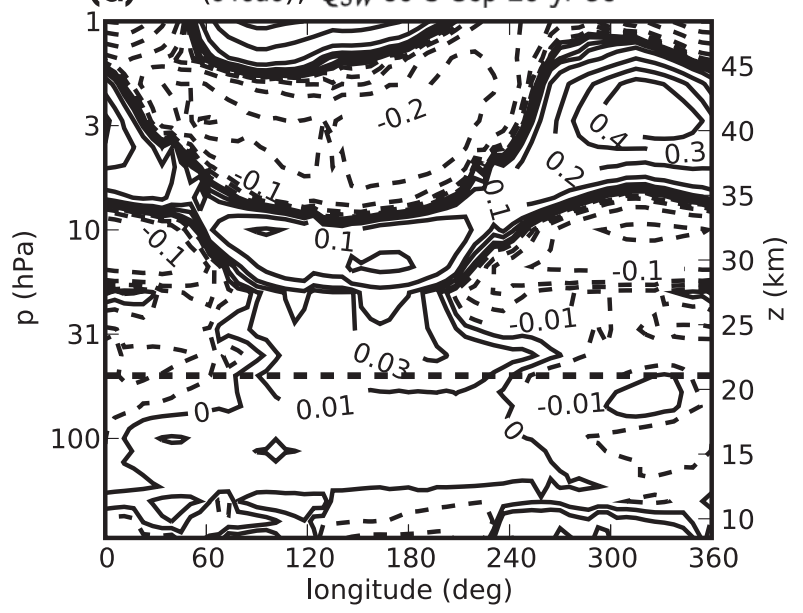

FIG. 7. (a) Daily mean temperature vs longwave heating rate for all longitudes and years at $50 \mathrm{hPa}, 80^{\circ} \mathrm{S}$ on $26 \mathrm{Sep}$. Data points for model year 38 are emphasized. Daily mean, zonally asymmetric (b) temperature, (c) longwave heating rate, and (d) shortwave heating rate on $26 \mathrm{Sep}$ at $80^{\circ} \mathrm{S}$ for model year 38 . The horizontal dashed line indicates $50 \mathrm{hPa}$. Shortwave heating anomalies are computed from the climatological mean at each 6-h model output and averaged, and then the zonal mean is subtracted to reduce aliasing.

The values determined here agree well with those determined using similar approaches with observed temperature and ozone fields. Ghazi et al. (1985) found shortwave damping of $0.12 \pm 0.06$ day $^{-1}$ at $1 \mathrm{hPa}$ computed from three days of data in the late winter near the vortex edge, falling to $0.02 \pm 0.02 \mathrm{day}^{-1}$ at $2 \mathrm{hPa}$, consistent with the gradients near $60^{\circ}$ found here. Equatorial solstice photochemical damping rates are also comparable with the scale-dependent rates computed by Haigh (1985), lying between the damping rates computed for uniform $(2 \pi / n=\infty)$ and short-wavelength $(2 \pi / n \approx$ $7.5 \mathrm{~km}$ ) perturbations. The meridional structure near the stratopause is also in close agreement. Pawson et al. (1992) found photochemical damping of approximately equal strength, though the region of transport-induced positive correlations was not identified.
The photochemical damping is a significant additional source of damping in the upper stratosphere and lower mesosphere. The total effective radiative damping $\left(\alpha_{\mathrm{LW}}+\alpha_{\mathrm{SW}}\right)$ is shown in Fig. 10. At the peak in longwave damping near the tropical stratopause, the daily zonal mean photochemical damping contributes an additional $30 \%$; this rises to nearly $50 \%$ of the radiative damping in the extratropics and is roughly equal to the longwave damping above the summer Antarctic. Significant hemispheric asymmetry is apparent at the tropical stratopause near solstice conditions as a result of the stronger photochemical damping in the summer hemisphere. The negative damping in the polar summer lower stratosphere is smaller in magnitude than the positive longwave damping. In the Antarctic spring, however, there is effectively no radiative damping as 
(a)

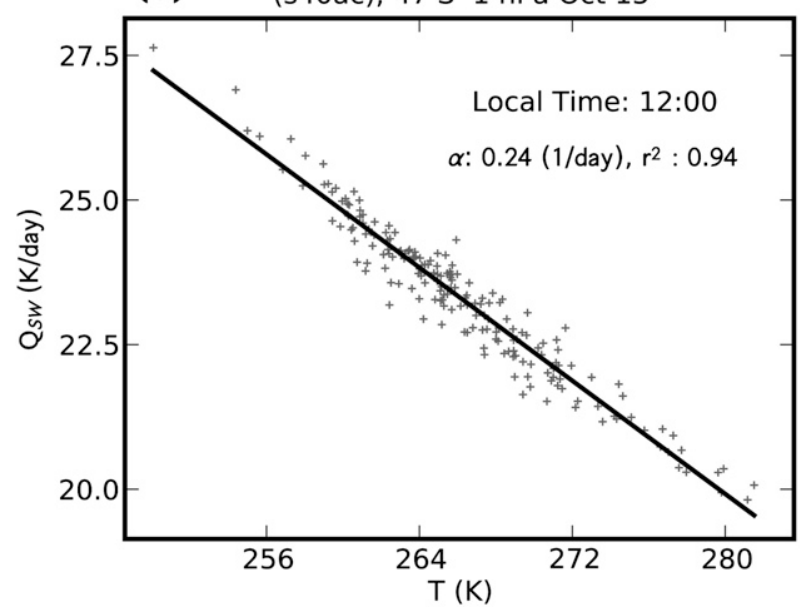

(c)

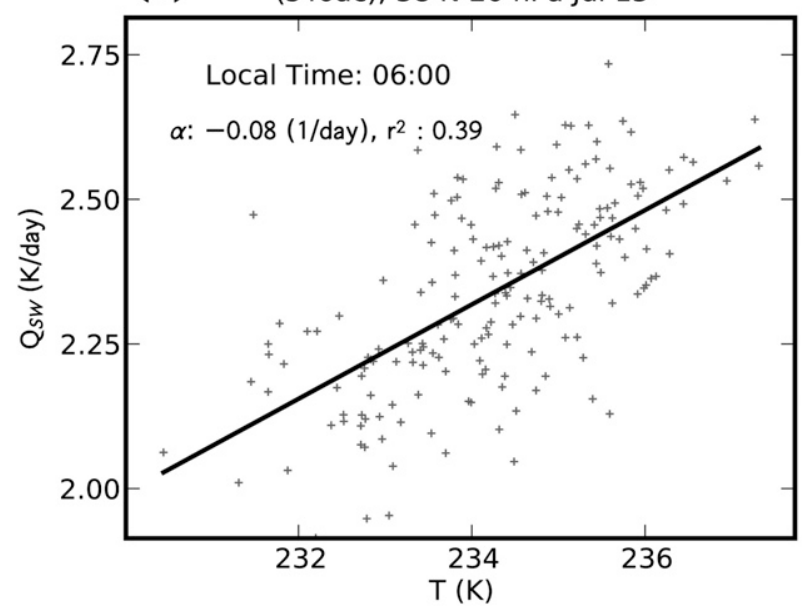

(b) (s40ac); $47 \mathrm{~S} 1 \mathrm{hPa}$ Oct 15

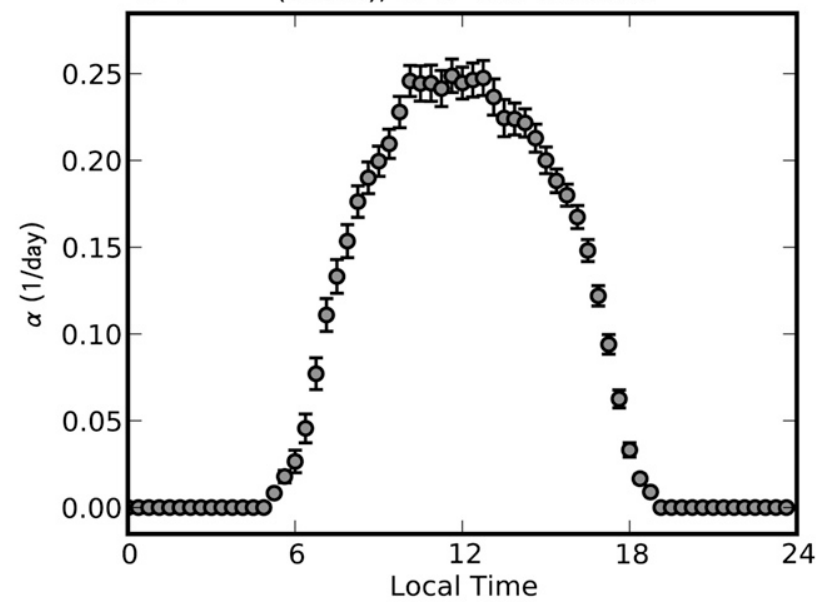

(d) (s40ac); 58 N 20 hPa Jul 15

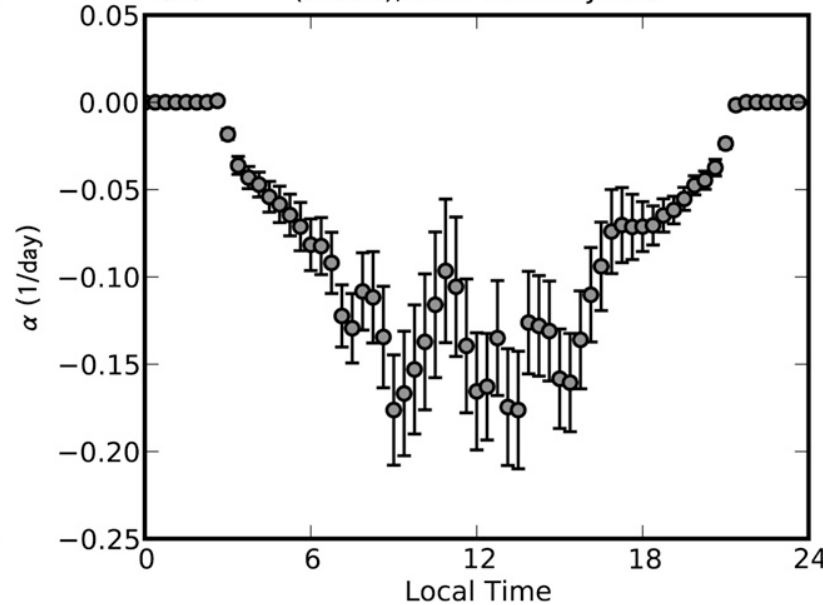

FIG. 8. Regression of shortwave heating rate against temperature at (a),(b) $47^{\circ} \mathrm{S}, 1 \mathrm{hPa}$ on 15 Oct and at (c),(d) $58^{\circ} \mathrm{N}, 20 \mathrm{hPa}$ on $15 \mathrm{Jul}$. (left) Scatterplots and fitted relationship for each day at the indicated local time, and (right) dependence of the damping rate on local time. Estimates of the uncertainty (at the $95 \%$ significance level) are indicated by the error bars in (b) and (d).

a result of the additional nonlocal shortwave effects (Fig. 7d).

\section{Estimating radiative-photochemical equilibrium temperature}

The quality of the empirical fits throughout much of the modeled middle atmosphere suggests the possibility of their use in computing a radiative-photochemical equilibrium temperature $T_{r}^{*}$ toward which the linearized radiative parameterizations of CMAM are effectively relaxing:

$$
\begin{aligned}
T_{r}^{*} & =[\bar{T}]_{c}+\frac{1}{\alpha}\left(\left[\overline{Q_{\mathrm{LW}}}\right]_{c}+\left[\overline{Q_{\mathrm{SW}}}\right]_{c}\right), \\
\alpha & = \begin{cases}\alpha_{\mathrm{LW}}+\alpha_{\mathrm{SW}} & \text { if } \alpha_{\mathrm{SW}}>0, \\
\alpha_{\mathrm{LW}} & \text { otherwise. }\end{cases}
\end{aligned}
$$

Since the negative damping rates are a result of transport, only the photochemical component of the shortwave damping rates is included. This estimate of $T_{r}^{*}$ is shown for 15 January in Fig. 11. Also shown for comparison is a computation from a time-marched computation of true radiative-photochemical equilibrium (Fels 1985). Between 10 and $0.1 \mathrm{hPa}$ and south of about $45^{\circ} \mathrm{N}$, where the effects of dynamics on zonal mean temperatures are small, the linear estimate matches the computation of Fels (1985) closely. The extremely cold radiative equilibrium in the polar vortex, however, is not captured. This failure is not solely a result of the curvature of the Planck function (though it is clear from Figs. 2e,f that linear extrapolation will overestimate the sensitivity of the heating rates and thus the radiative equilibrium temperature). It is also a result of the balance in radiative equilibrium between the emission and absorption of longwave 

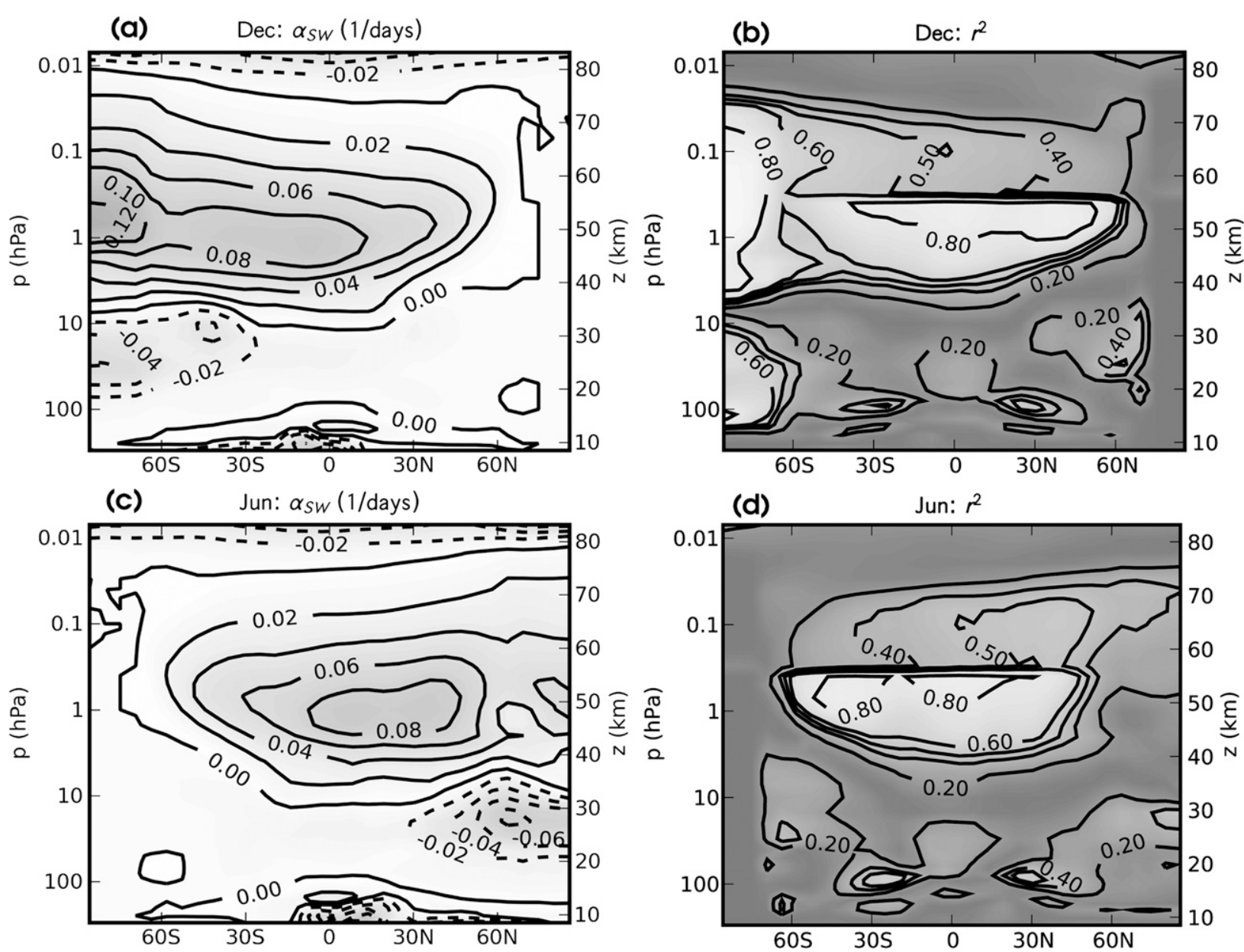

FIG. 9. Daily averaged shortwave damping rates and coefficients of determination for (a),(b) December and (c),(d) June. A day-night mask is applied when averaging the coefficients of determination. Contour spacing for the shortwave damping rates is 0.02 day ${ }^{-1}$. Contours for the coefficients of determination are shown at $0.2,0.4,0.5,0.6$, and 0.8 .

radiation, due to the absence of shortwave heating, which implies the importance of nonlocal contributions. Indeed, the cool-to-space approximation predicts that longwave radiation cools at any temperature above absolute zero. In the absence of shortwave heating, the assumption of locality fails rather more profoundly than that of linearity in predicting the true photochemical-radiative equilibrium temperature. In the real atmosphere, longwave radiation always cools in the polar vortex in order to balance dynamical heating, so the local assumption holds reasonably well (as indicated by Fig. 6c). The linear estimate also overestimates true radiative equilibrium in the upper summer mesosphere, where nonlocal effects (from the warm stratopause below) are again important.

\section{Discussion and conclusions}

The assumption that radiative heating rates in the middle atmosphere respond linearly to changes in local temperature is satisfied sufficiently well in most cases to define an effective relaxation rate that varies with height, latitude, and season. This effective damping rate can be usefully diagnosed with simple linear regression models. This has been shown in the stratosphere by previous studies combining observed or reanalyzed temperatures and trace gas concentrations with offline radiative transfer models (Pawson et al. 1992; Newman and Rosenfield 1997). Here we have explored this relationship more thoroughly in a chemistry-climate model with comprehensive physical, radiative, and chemical parameterizations. The model broadly confirms this result throughout the stratosphere and mesosphere, where the linear model explains more than $90 \%$ of the variance in longwave heating rates through much of the extratropical stratosphere and more than $80 \%$ in the mesosphere and tropical upper stratosphere. Where it is well specified, the regression model diagnoses effective longwave damping rates in these 40 -yr time-slice runs to a sampling error of 

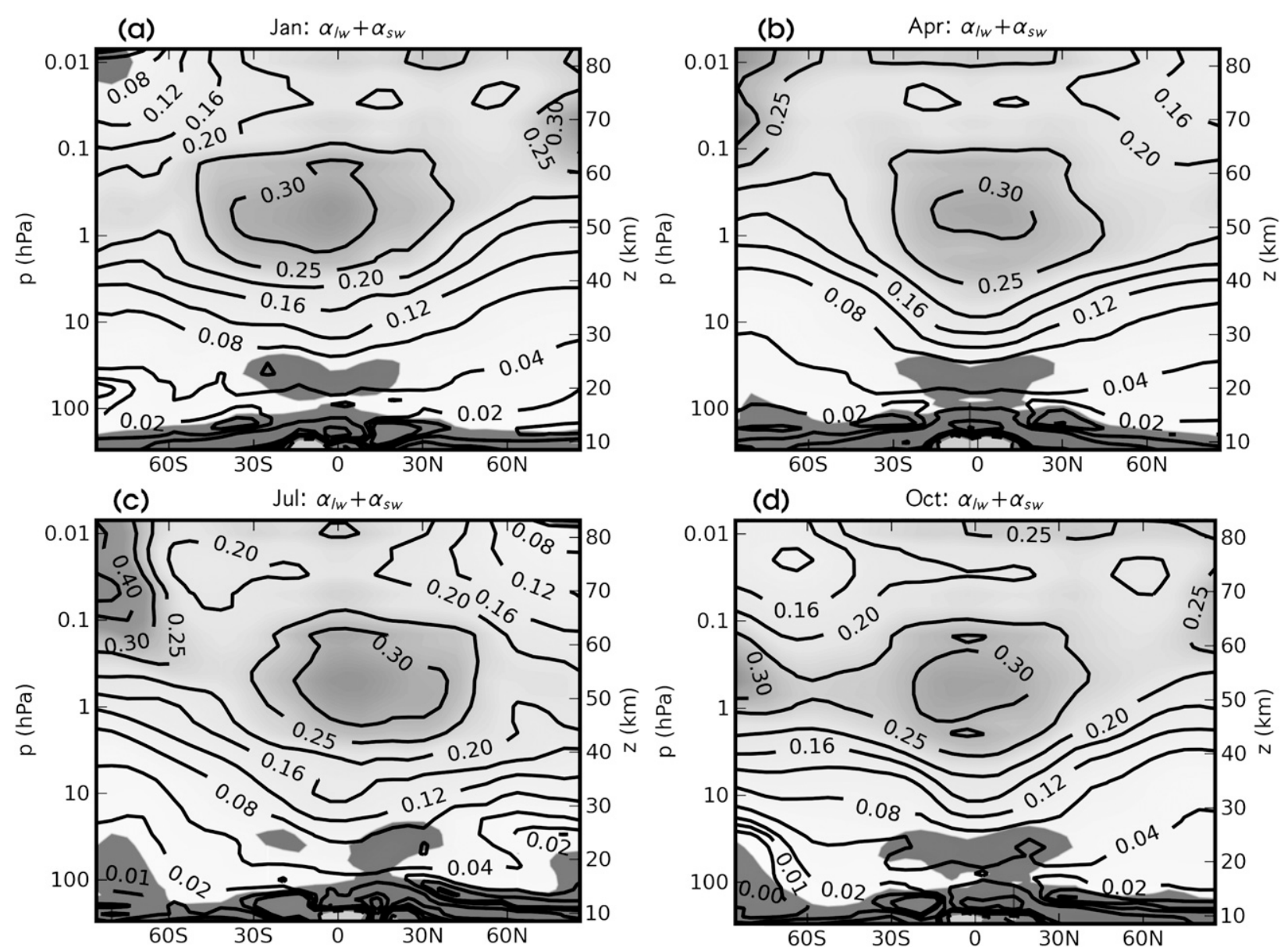

FIG. 10. Sum of monthly mean longwave and shortwave damping rates $\left(\right.$ day $^{-1}$ ) for (a) January, (b) April, (c) July, and (d) October. Dark shading is as in Fig. 4. Note the zero contour in the lower Antarctic stratosphere in (d).

about $5 \%$. The regressed damping rates agree roughly with the parameterization of Fels (1982) but suggest that changes in the spectrum of vertical length scales in temperature disturbances generate a greater meridional and seasonal variation than would be implied by the dependence on background temperatures alone.

Shortwave heating rates are also well modeled by a simple, diurnally averaged linear damping rate near the stratopause where the variance explained is greater than 0.8 . In this region they provide a significant correction to longwave damping of temperature anomalies. Positive correlations with the temperature are also found in the lower summer stratosphere as a result of eddy transport of ozone, though they are less well characterized by a climatologically specified effective damping rate.

The linear regression model also provides a means of estimating the climatological radiative-photochemical equilibrium temperature in the middle atmosphere. The calculation for the runs analyzed here agrees well with the explicit calculation of Fels (1985) where climatological temperatures do not depart drastically from the equilibrium, but it significantly overestimates the equilibrium temperature in the polar night.

Understanding where the regression model fails in the chemistry-climate model runs analyzed here gives insight into three ways in which a simple Newtonian cooling parameterization fails to capture the response of radiative heating rates to temperature perturbations.

First, in the lower tropical stratosphere, temperature perturbations with small vertical scales are damped significantly more strongly than more typical perturbations with larger vertical scales (Fig. 1c). The details of this particular failure are almost certainly sensitive to the presence of a QBO (which is absent in these runs); however, the presence of a broader spectrum of vertical scales in the tropics suggests that the neglect of the scale dependence of damping rates there is of more general concern.

Second, in the lower Antarctic vortex (particularly in late winter and spring), the assumption of locality again 

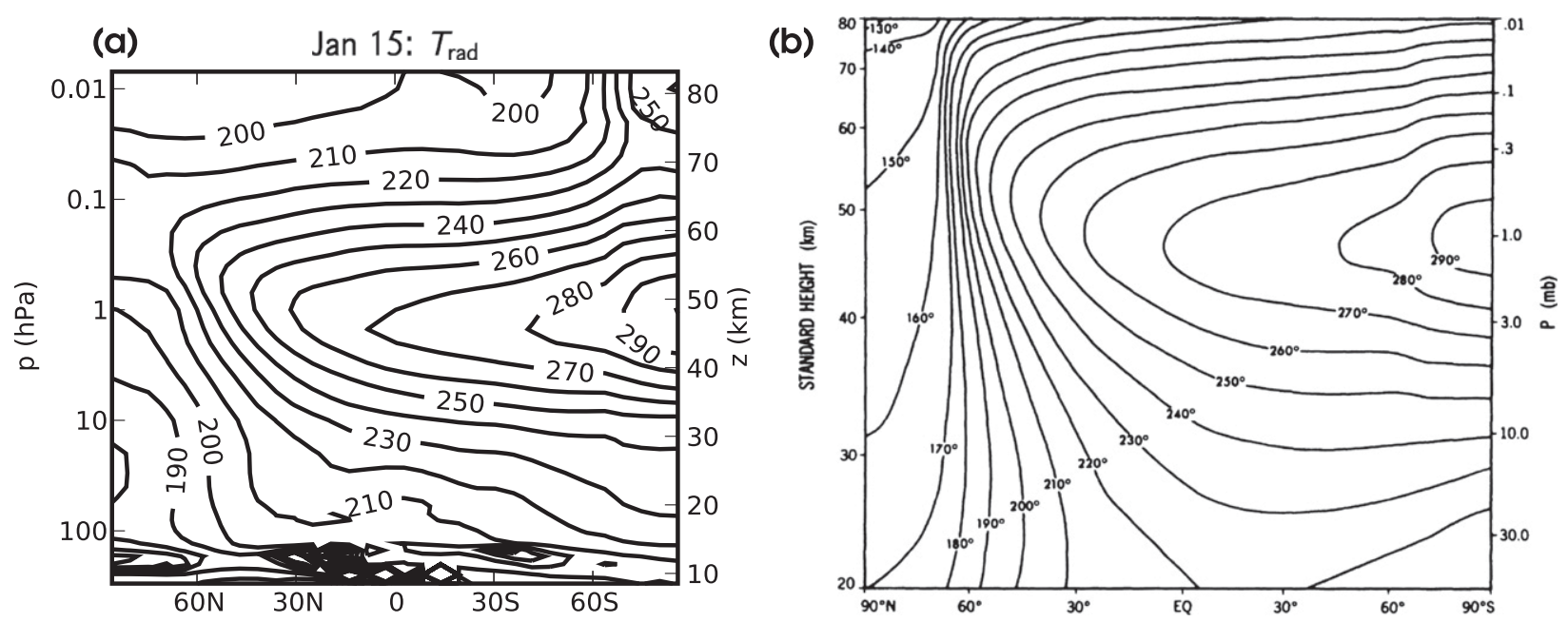

FIG. 11. (a) Linear estimate of the radiative equilibrium temperature for 15 Jan. (b) Radiative-photochemical equilibrium on the same date computed by Fels (1985) by time stepping through the seasonal cycle [reprinted from Fels (1985) with permission from Elsevier]. Note the nonstandard vertical scale of (b) and that the Southern Hemisphere is to the right in both panels.

fails. Here, however, it fails in a way that cannot be explained by the scale-dependent parameterization. The variance in temperatures above the relatively stable Antarctic vortex is large enough to dominate the local influence on longwave heating rates. Moreover, the behavior of the longwave radiation depends strongly on the state of the vortex from year to year. The assumption of an interannually constant, linear damping rate does not hold.

Third, the large climatological variance in temperatures near the edge of the polar vortex in both hemispheres leads to significant nonlinearity in the longwave heating rates as a result of the curvature of the Planck function. Dynamically, this may be the most important departure from the assumptions implicit in the use of an effective linear relaxation identified in this work, given that the linearized relaxation rate can vary by a factor of 2 near the vortex edge when a realistic parameterization of longwave heating rates is used.

Acknowledgments. The authors thank Victor Fomichev for helpful comments on this manuscript. Peter Hitchcock gratefully acknowledges support from the Walter Sumner Foundation and from the Japan Society for the Promotion of Science 2008 Summer Program, during which this work was initiated. The CMAM simulations were provided through the C-SPARC project supported by the Canadian Foundation for Climate and Atmospheric Sciences.

\section{REFERENCES}

Andrews, D. G., and M. E. McIntyre, 1978: Generalized EliassenPalm and Charney-Drazin theorems for waves on axisymmetric mean flows in compressible atmospheres. J. Atmos. Sci., 35, 175-185.
— J. R. Holton, and C. B. Leovy, 1987: Middle Atmosphere Dynamics. Academic Press, 489 pp.

Bresser, G., A. J. L. Manning, S. Pawson, and C. D. Rodgers, 1995: A new parameterization of scale-dependent radiative rates in the stratosphere. J. Atmos. Sci., 52, 4429-4447.

Craig, R. A., and G. Ohring, 1958: The temperature dependence of ozone radiational heating rates in the vicinity of the mesopeak. J. Meteor., 15, 59-62.

de Grandpré, J., S. R. Beagley, V. I. Fomichev, E. Griffioen, J. C. McConnell, A. S. Medvedev, and T. G. Shepherd, 2000: Ozone climatology using interactive chemistry: Results from the Canadian Middle Atmosphere Model. J. Geophys. Res., 105, 26 475-26 491.

Dickinson, R. E., 1973: Method of parameterization for infrared cooling between altitudes of 30 and 70 kilometers. J. Geophys. Res., 78, 4451-4457.

Eyring, V., and Coauthors, 2006: Assessment of temperature, trace species, and ozone in chemistry-climate model simulations of the recent past. J. Geophys. Res., 111, D22308, doi:10.1029/ 2006JD007327.

Fels, S. B., 1982: A parameterization of scale-dependent radiative damping rates in the middle atmosphere. J. Atmos. Sci., 39, 1141-1152.

_ 1984: The radiative damping of short vertical scale waves in the mesosphere. J. Atmos. Sci., 41, 1755-1764.

_-1985: Radiative-dynamical interactions in the middle atmosphere. Advances in Geophysics, Vol. 28, Academic Press, 277-300.

Fomichev, V. I., and J.-P. Blanchet, 1995: Development of the new $\mathrm{CCC} / \mathrm{GCM}$ longwave radiation model for extension into the middle atmosphere. Atmos.-Ocean, 33, 513-531.

,-- , and D. S. Turner, 1998: Matrix parameterization of the $15 \mu \mathrm{m} \mathrm{CO}_{2}$ band cooling in the middle and upper atmosphere for variable $\mathrm{CO}_{2}$ concentration. J. Geophys. Res., 103,11 505-11 528.

- C. Fu, J. de Grandpré, S. R. Beagley, V. P. Ogibalov, and J. C. McConnell, 2004: Model thermal response to minor radiative energy sources and sinks in the middle atmosphere. J. Geophys. Res., 109, D19107, doi:10.1029/2004JD004892.

Garcia, R. R., and B. A. Boville, 1994: "Downward control" of the mean meridional circulation and temperature distribution of the polar winter stratosphere J. Atmos. Sci., 51, 2238-2245. 
Ghazi, A., P.-H. Wang, and M. P. McCormick, 1985: A study on radiative damping of planetary waves utilizing stratospheric observations. J. Atmos. Sci., 42, 2032-2042.

Gille, J. C., and L. V. Lyjak, 1986: Radiative heating and cooling rates in the middle atmosphere. J. Atmos. Sci., 43, 22152229.

Haigh, J. D., 1985: A fast method for calculating scale-dependent photochemical acceleration in dynamical models of the stratosphere. Quart. J. Roy. Meteor. Soc., 111, 1027-1038.

Hartmann, D. L., 1981: Some aspects of the coupling between radiation, chemistry, and dynamics in the stratosphere. J. Geophys. Res., 86, 9631-9640.

Holton, J. R., and R. S. Lindzen, 1972: An updated theory for the quasi-biennial cycle of the tropical stratosphere. J. Atmos. Sci., 29, 1076-1080.

_ Sci., 33, 2218-2225.

Kiehl, J. T., and S. Solomon, 1986: On the radiative balance of the stratosphere. J. Atmos. Sci., 43, 1525-1534.

Leovy, C. B., 1984: Infrared radiative exchange in the middle atmosphere in the 15-micron band of carbon dioxide. Dynamics of the Middle Atmosphere, J. R. Holton and T. Matsuno, Eds., Terrapub, 355-366.

Marsh, D., A. Smith, G. Brasseur, M. Kaufmann, and K. Grossmann, 2001: The existence of a tertiary ozone maximum in the highlatitude middle mesosphere. Geophys. Res. Lett., 28, 45314534.

McLandress, C., 2002: The seasonal variation of the propagating diurnal tide in the mesosphere and lower thermosphere. Part I: The role of gravity waves and planetary waves. J. Atmos. Sci., 59, 893-906.

Mlynczak, M. G., C. J. Mertens, R. R. Garcia, and R. W. Portmann, 1999: A detailed evaluation of the stratospheric heat budget 2 .
Global radiation balance and diabatic circulations. J. Geophys. Res., 104, 6039-6066.

Nathan, T. R., E. C. Cordero, and L. Li, 1994: Ozone heating and the destabilization of traveling waves during summer. Geophys. Res. Lett., 21, 1531-1534.

Newman, P. A., and J. Rosenfield, 1997: Stratospheric thermal damping times. Geophys. Res. Lett., 24, 433-436.

Pawson, S., R. S. Harwood, and J. D. Haigh, 1992: A study of the radiative dissipation of planetary waves using satellite data. J. Atmos. Sci., 49, 1304-1317.

Polvani, L. M., and P. J. Kushner, 2002: Tropospheric response to stratospheric perturbations in a relatively simple general circulation model. Geophys. Res. Lett., 29, 1114, doi:10.1029/ 2001 GL014284.

Rodgers, C. D., and C. D. Walshaw, 1966: The computation of infrared cooling rate in planetary atmospheres. Quart. J. Roy. Meteor. Soc., 92, 67-92, doi:10.1002/qj.49709239107.

Sasamori, T., and J. London, 1966: The decay of small temperature perturbations by thermal radiation in the atmosphere. J. Atmos. Sci., 23, 543-554.

Scinocca, J. F., N. A. McFarlane, M. Lazare, J. Li, and D. Plummer, 2008: The CCCma third-generation AGCM and its extensions into the middle atmosphere. Atmos. Chem. Phys., 8, 7055-7074.

Taguchi, M., T. Yamaga, and S. Yoden, 2001: Internal variability of the troposphere-stratosphere coupled system simulated in a simple global circulation model. J. Atmos. Sci., 58, 31843203.

Waugh, D. W., and V. Eyring, 2008: Quantitative performance metrics for stratospheric-resolving chemistry-climate models. Atmos. Chem. Phys., 8, 5699-5713.

Zhu, X., 1993: Radiative damping revisited: Parameterization of damping rate in the middle atmosphere. J. Atmos. Sci., 50, 3008-3021. 